\title{
STEREOLOGICAL ANALYSIS OF THE COCHLEAR NUCLEI OF MONKEY (MACACA FASCICULARIS) AFTER DEAFFERENTATION
}

\author{
AnA M INSAUSTI ${ }^{1}$, RICARdo InSAUSTI $^{2}$, LUIS M CRUZ-ORIVE ${ }^{1}$ AND MANUEL MANRIQUE ${ }^{3}$ \\ ${ }^{1}$ Department of Mathematics, Statistics and Computation, Faculty of Sciences, University of Cantabria, \\ E-39005 Santander, Spain, ${ }^{2}$ Department of Anatomy, University of Castilla La Mancha, School of Medicine, \\ E-02071 Albacete, Spain, ${ }^{3}$ Department of Otorhinolaryngology, School of Medicine, University of Navarra, \\ E-31080 Pamplona, Spain \\ (Accepted April 19, 2000)
}

\begin{abstract}
The cochlear nuclei $(\mathrm{CN})$ in the brainstem receive the input signals from the inner ear through the cochlear nerve, and transmit these signals to higher auditory centres. A variety of lesions of the cochlear nerve cause deafness. As reported in the literature, artificial removal of auditive input, or 'deafferentation', induces structural alterations in the $\mathrm{CN}$. The purpose of this study was to estimate a number of relevant stereological parameters of the $\mathrm{CN}$ in control and deafferented Macaca fascicularis monkeys.
\end{abstract}

Keywords: Cavalieri estimator, cochlear nuclei, deafferentation, glia, nonhuman primate, stereology, vertical slices.

\section{INTRODUCTION}

The cochlear nuclei $(\mathrm{CN})$ are responsible for the first step in the central auditory pathways, as they receive the input from the inner ear through the cochlear nerve and project it to higher auditory centres. A number of pathological events may affect the cochlear nerve and cause an important loss of input, which, in turn, may induce changes in the inner structure of the $\mathrm{CN}$, and hence partial or total deafness. $\mathrm{CN}$ shrinkage and neuron loss after deafferentation have been reported qualitatively (Hardie et al., 1999, and references therein). The glial component of the $\mathrm{CN}$, and mainly the astrocyte population, has hitherto not been assessed quantitatively, despite of its important role in the maintenance of neural circuits (ValderramaCanales et al., 1993).

The aim of this study was to quantify the changes induced in the $\mathrm{CN}$ of the cynomolgus monkey (Macaca fascicularis) after chronic lesion of the cochlear nerve.

We used a vertical slices design (Gokhale,1990; Batra et al., 1995) to estimate the total volume of the $\mathrm{CN}$ and the total length of astrocyte processes in control and deafferented monkeys. The total number of neurons in the $\mathrm{CN}$ was estimated by a conventional combination of Cavalieri slices and the optical disector (e.g.West et al., 1996) in another two groups of control and deafferented monkeys from a related study.

\section{MATERIAL AND METHODS}

\section{ANIMALS, SURGERY AND PREPARATION OF THE MATERIAL}

A total of 21 Macaca fascicularis monkeys were used. In a first study aimed at estimating neuron number in the $\mathrm{CN}, 6$ controls and 6 deafferented animals were used. In a second study involving the vertical design, 4 controls and 5 deafferented animals were used. Animals were housed individually, and all procedures were carried out according to the European Union rules (86/609/CEE).

Each animal underwent bilateral surgery under aseptic conditions with general anesthesia. After a translabyrinthine approach, the cochlear nerve was sectioned extradurally, and a small portion was removed to avoid contact with the terminals. After three months, animals were euthanized with an overdose of barbiturates and perfused transcardially with a solution of $4 \%$ paraformaldehyde in phosphate buffer ( $\mathrm{pH} \mathrm{7.4).} \mathrm{The} \mathrm{brain} \mathrm{was} \mathrm{then} \mathrm{placed} \mathrm{in}$ cryoprotective solution. For additional details see Insausti et al. (1999). 


\section{VERTICAL SLICES DESIGN TO ESTIMATE ASTROCYTE PROCESS LENGTH}

In each of the 4 controls both halves of the $\mathrm{CN}$ were analyzed, whereas in 4 of the 5 deafferented animals only one half was selected with probability $1 / 2$ and analyzed. For each animal, the target parameters were:

$V($ nuc $)=$ Total volume of the union of left and right $\mathrm{CN}$.

$L($ proc $)=$ Total length of the astrocyte processes in the $\mathrm{CN}$.

$L$ (proc) was estimated via the ratio $L_{V}$ (proc, nuc $)=L($ proc $) / V($ nuc $)$ as follows:

$$
L(\text { proc })=L_{V}(\text { proc }, \text { nuc }) \cdot V(\text { nuc }), m .
$$

The ratio-unbiased estimation of $L_{V}$ (proc, nuc) requires relative isotropy between specimen and test probes. We adopted a vertical slices design (Gokhale, 1990; Batra et al., 1995), see Fig. 1. The brainstem was separated from the brain and halved by a midsagittal cut (Fig. 1a). The artificial sagittal face of each half was adopted as horizontal plane (HP) and the long axis of the brainstem was adopted as origin of vertical slice orientation angle $\phi$, (Fig. 1b). For the first animal in a group of $n$ animals, $\phi=\phi_{1}$ was uniform random in the interval $\left(0,180^{\circ} / n\right)$; for the $i$ th animal, $\phi=\phi_{i}=\phi_{i-1}+180^{\circ} / n,(i=2,3, \ldots, n)$. Thus, only one random number was needed per group. The companion $\mathrm{CN}$ side (whenever available) of the $i$ th animal was cut at an angle $\phi_{i}+90$ respecting the angle origin. At each angle, a series of vertical slices encompassing the $\mathrm{CN}$ side entirely was cut. Two different slice thicknesses were used, namely 30 and $60 \mu \mathrm{m}$,.in the order shown in Fig. 1c. The first $60 \mu \mathrm{m}$ thick slice in each period was stained

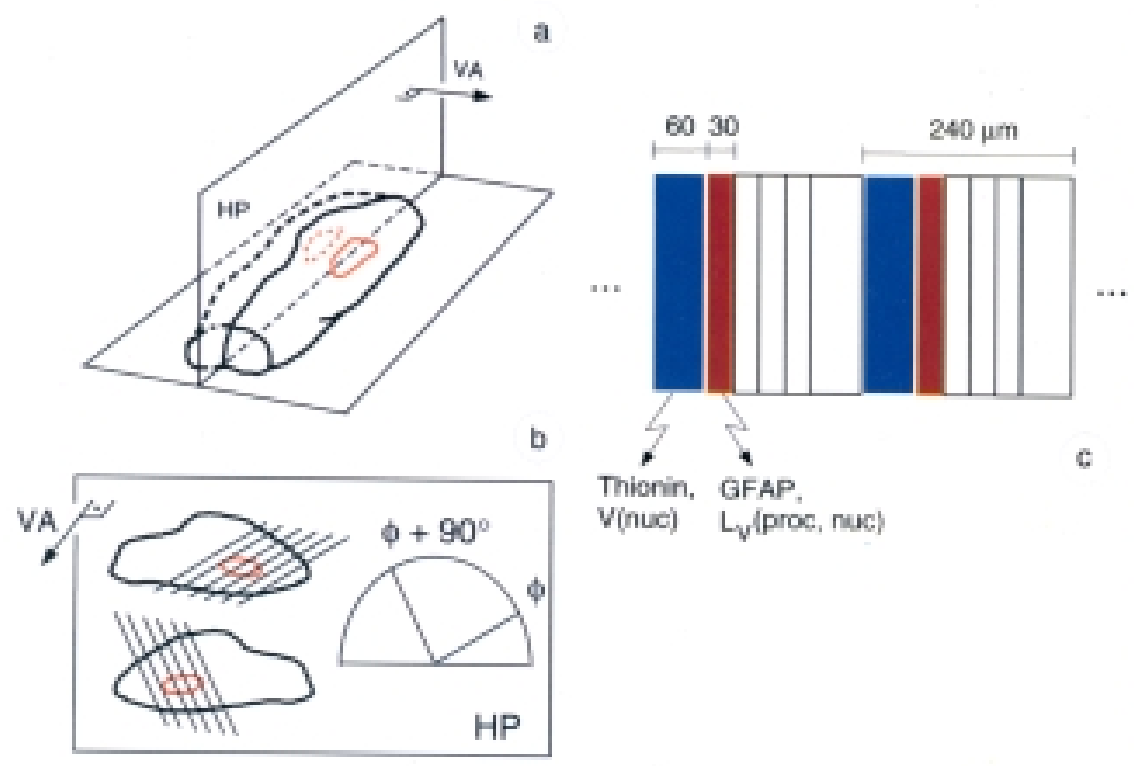

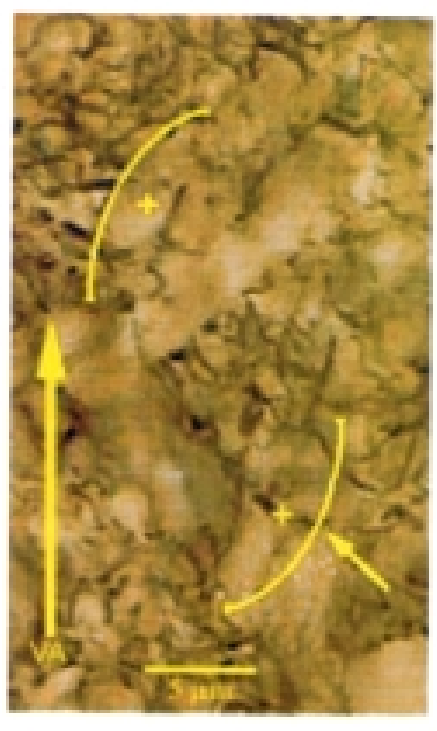

Vertical $30 \mu \mathrm{m}$ thick slice (GFAP stained)

Fig. 1. Vertical slices design. (a) Sketch of brainstem of monkey halved by a midsagittal cut. The two CN sides are outlined in red. $H P=$ adopted horizontal plane, $V A=$ vertical axis. (b) Stack of vertical slices (normal to the paper) at a uniform random angle $\phi$ for one $C N$ side, and at $\phi+90^{\circ}$ for the other (arrowheads in HP: angle origins). (c) Two periods of the vertical stacks. (d) A portion of a vertical slice to estimate $L_{V}$, with two cycloid arcs and two test points superimposed on it. The latter are kept unchanged by the CAST-Grid as the slice is scanned up-down to record the relevant intersections (arrowhead) between astrocyte processes and cycloids. 
with thionin to easily highlight the delineation of the $\mathrm{CN}$; these slices were destined to estimate $V($ nuc) by the Cavalieri formula (e.g. Howard and Reed, 1998):

$$
\hat{V}(\text { nuc })=T \cdot \frac{a}{p} \cdot \sum P(\text { nuc }) \mathrm{mm}^{3},
$$

where $T=0.240 \mathrm{~mm}$, and $\sum P($ nuc $)$ is the total number of points counted in the $\mathrm{CN}$ with a test system of $a / p=0.29 \mathrm{~mm}^{2}$ area per test point, corrected for linear magnification (x25, LM). $\sum P($ nuc) ranged from 85 to 147 per $\mathrm{CN}$ side. The next $30 \mu \mathrm{m}$ thick slice was stained with a specific antibody against glial fibrillary.acidic protein (GFAP) to highlight the astrocytic processes. Every fourth of the GFAP slices was destined to estimate $L_{V}$ (proc, nuc) by intersection counting with properly oriented cycloids (Fig. 1d). The $\mathrm{CN}$ boundaries were identified by direct reference to the adjacent $60 \mu \mathrm{m}$ thick thionin slice. Thus, for each animal,

$$
\hat{L}_{V}(\text { proc }, \text { nuc })=\frac{2}{t} \cdot \frac{p}{l} \cdot \frac{\sum I(\text { proc })}{\sum P(\text { nuc })} \mathrm{m} / \mathrm{mm}^{3},
$$

where $t=0.030 \mathrm{~mm}$ is the section thickness and $\mathrm{P} / l=41.08 \mathrm{~mm}^{-1}$ is the ratio of test point number to cycloid test line lenght corrected for magnification whereas $\sum I$ (proc) represents the total number of intersections between the projections of astrocyte processes in the $\mathrm{CN}$ inside the vertical slices and the cycloid test lines, and $\sum P($ nuc) the number of test points counted in all systematic quadrats subsampled (at x1000 by LM) in all vertical slices. $\sum I$ (proc) ranged from 121 to 318 , whereas $\sum P($ nuc) ranged from 12 to 38 in different $\mathrm{CN}$ sides. Slice subsampling and point and intersection counting were carried out with the aid of the CAST-Grid system (Olympus Denmark, DK-Albertslund).

\section{CAVALIERI DESIGN AND OPTICAL DISECTORS TO ESTIMATE NEURON NUMBER}

For each of the 6 controls and 6 deafferented animals the target parameters were $V($ nuc), as before, and:

- $\quad N($ neu $)=$ Total number of neurons in the CN.

Both $\mathrm{CN}$ sides of each animal were exhaustively sectioned into stacks composed as indicated in Fig. 1c. The sections, however, were always perpendicular to the long axis of the brainstem - hence they were not vertical but Cavalieri slices, see Baddeley et al. (1986). Only the $60 \mu \mathrm{m}$ thick thionin slices were used. $V$ (nuc) was estimated as before (Eq. 2), either with $a / p=0.103 \mathrm{~mm}^{2}$, or with $0.072 \mathrm{~mm}^{2}$, to suit different CN sizes; between 161 and 387 test points were counted per $\mathrm{CN}$ side. Further, $N($ neu) was estimated indirectly via

$$
N(\text { neu })=N_{V}(\text { neu, nuc }) \cdot V(\text { nuc })
$$

The ratio $N_{V}$ (neu, nuc) was estimated by systematic optical disectors on each Cavalieri slice. For each systematic quadrat, one central optical disector with an associated test point was generated with the aid of the CAST-Grid system. Two different optical disector areas, namely $a / p=1318 \mu \mathrm{m}^{2}$ and $1647 \mu \mathrm{m}^{2}$ (corrected for a final linear magnification of 1000) were used to suit different neuron numerical densities in different CN's. The uncorrected disector height was always $10 \mu \mathrm{m}$, measured with the microcator of the CAST-Grid system. The mean final section thickness, also measured with the microcator, was $34 \mu \mathrm{m}$. Since the initial section thickness was $60 \mu \mathrm{m}$, the disector height corrected for tissue shrinkage was $h=10 \times 60 / 34=17.6 \mu \mathrm{m}$. The relevant ratio was estimated as

$$
\hat{N}_{V}(\text { neu, nuc })=\frac{\sum Q^{-}(\text {neu })}{h \cdot(a / p) \cdot \sum P(\text { nuc })} \mu \mathrm{m}^{-3},
$$

where $\sum Q^{-}$(neu) represents the total number of neurons counted in a $\mathrm{CN}$ in all disectors, and $\sum P($ nuc) the total number of disector-associated test points hitting the CN. $\sum Q^{-}$(neu) varied between 166 and 367 , whereas $\sum P($ nuc) varied between 161 and 387 per $\mathrm{CN}$ side.

\section{RESULTS AND CONCLUSIONS}

The results are given in Fig. 2. For the deafferented group from the vertical design, whenever a single $\mathrm{CN}$ side was available the final estimate of $V($ nuc $)$ and of $L$ (proc) for the whole $\mathrm{CN}$ was twice the corresponding estimate from the available side. 

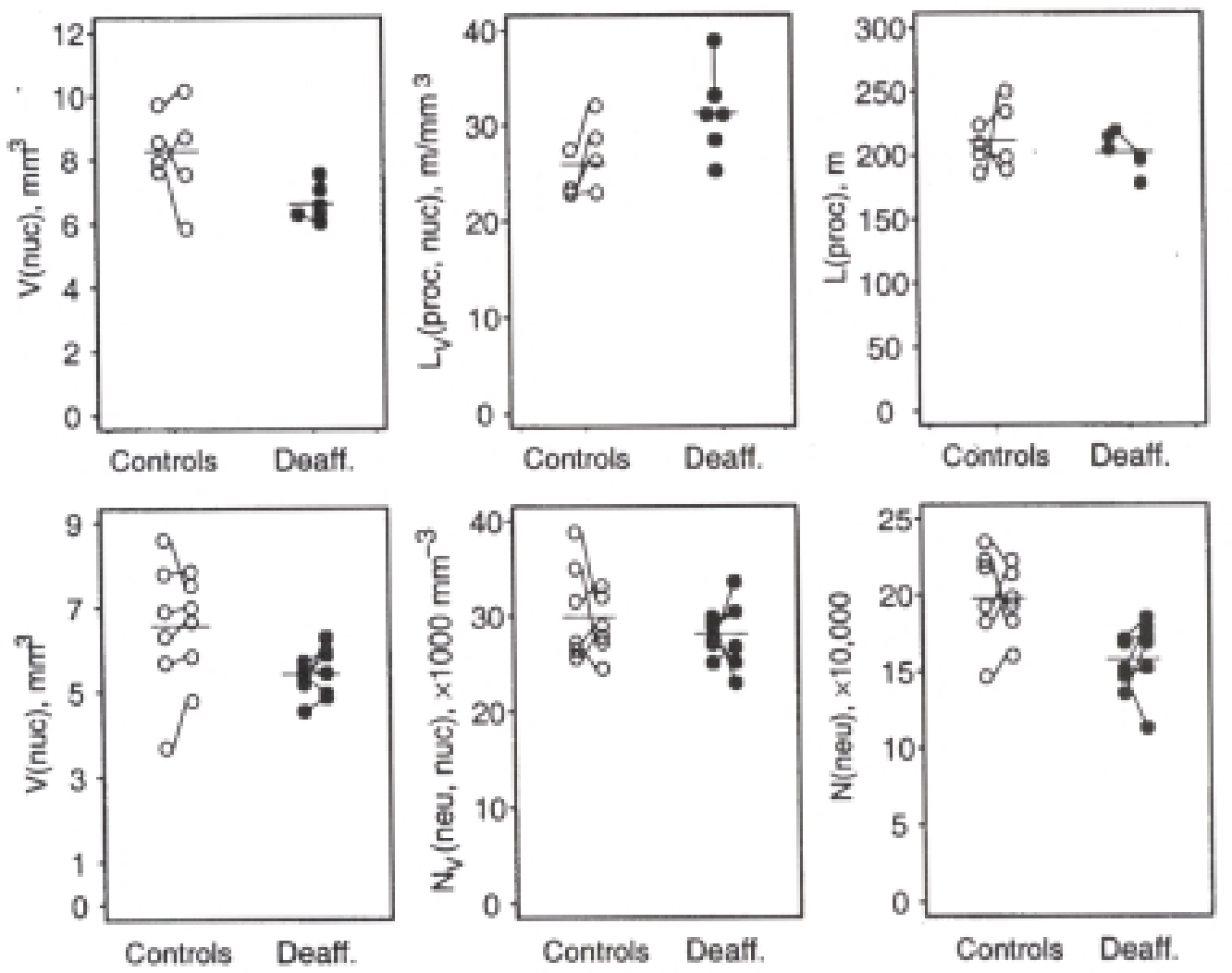

Fig. 2. Results of the experiments. Dipoles correspond to the two sides(whenever available) of a same CN. Horizontal segments represent group means.

Confidence intervals for the mean group differences of relevant parameters are displayed in

Table 1 below. For a generic parameter $Z$, the notation " $\Delta Z$ " means

Table1. Results of the two designs described in this paper. For explanations, see text.

\begin{tabular}{lllll}
\hline Design & Conf. level & $\Delta V($ nuc $), \mathrm{mm}^{3}$ & $\Delta V_{L}, \mu \mathrm{m}^{2}$ & $\Delta L$ (proc), m \\
\hline Vertical & $90 \%$ & $(0.65,5.56)$ & $(0.21,10.28)$ & $(-14.0,59.2)$ \\
slices & $95 \%$ & $(0.04,6.17)$ & $(-1.04,11.52)$ & $(-23.1,68.2)$ \\
\hline Design & Conf. level & $\Delta V($ nuc $), \mathrm{mm}^{3}$ & $\Delta V_{N}($ nuc $), \mu \mathrm{m}^{3}$ & $\Delta N($ neu $),\left(10^{3}\right)$ \\
\hline Cavalieri & $90 \%$ & $(0.08,4.45)$ & $(-5100,1700)$ & $(35,125)$ \\
$\&$ optical disectors & $95 \%$ & $(-0.42,4.95)$ & $(-5900,2500)$ & $(25,135)$ \\
\hline
\end{tabular}

"mean $(Z)$ in the control population - mean $(Z)$ in the deafferented population". Further, $V_{L}=1 / L_{V}$ (proc, nuc) may be regarded as the mean cross-sectional area of a cylinder model for the space surrounding an astrocyte process. The group mean values of $V_{L}$ were $39.0 \mu \mathrm{m}^{2}$ (which corresponds to a circle of $7.0 \mu \mathrm{m}$ diameter, $\mathrm{SE}=0.2 \mu \mathrm{m}$ ) for the control group, and $33.7 \mu \mathrm{m}^{2}$ (circle of $6.5 \mu \mathrm{m}$ diameter, $\mathrm{SE}=0.2 \mu \mathrm{m}$ ) for the deafferented group. Likewise, $V_{N}=1 / N_{V}$ (neu, nuc) can be regarded as the mean volume of space available to a neuron. The group mean values of $V_{N}$ were $32900 \mu \mathrm{m}^{3}$ (corresponding to a sphere of $39.7 \mu \mathrm{m}$ diameter, $\mathrm{SE}=0.6 \mu \mathrm{m}$ ) for the control group and $34600 \mu \mathrm{m}^{3}$ (sphere of $40.4 \mu \mathrm{m}$ diameter, $\mathrm{SE}=0.5 \mu \mathrm{m}$ ) for the deafferented group.

The data reveal the following:

1. Deafferentation seems to induce a reduction in the volume of the $\mathrm{CN}$. 
2. The total length of astrocytic processes in the $\mathrm{CN}$ seems to be unaffected by deafferentation. It is noteworthy that this is the result of an increase in $L_{V}$ (proc, nuc) concomitant with a decrease in $V($ nuc) for each animal.

3. Deafferentation seems to reduce the total number of neurons in the $\mathrm{CN}$. This reduction obeys solely to the reduction in total $\mathrm{CN}$ volume, because $N_{V}$ (neu, nuc) was practically unaffected.

A preliminary report of some of the data was presented at the $X^{\text {th }}$ International Congress for Stereology, Melbourne, Australia, 1-4 November 1999.

\section{ACKNOWLEDGMENTS}

This work was supported by the University of Navarra Research TT Program, the Cochlear Company, the University of Cantabria, the Dirección General de Enseñanza Superior (DGES) Project No. PM97-0043 and Biomed II (E.U.) No. BMH4-CT97-2437.

\section{REFERENCES}

Baddeley AJ, Gundersen HJG, Cruz-Orive LM (1986). Estimation of surface area from vertical sections. J Microsc 142:259-76.
Batra S, König MF, Cruz-Orive LM (1995). Unbiased estimation of capillary length from vertical slices. J Microsc 178:152-9.

Gokhale AM (1990). Unbiased estimation of curve length in 3-D using vertical slices. J Microsc 159:133-41.

Hardie NA, Shepherd RK (1999). Sensorineural hearing loss during development: morphological and physiological response of the cochlea and auditory brain stem. Hearing Research 128:147-65.

Howard CV, Reed MG (1998). Unbiased Stereology. Three-dimensional measurement in microscopy. Oxford: Bios Sci Publ.

Insausti AM, Cruz-Orive LM, Jáuregui I, Manrique M, Insausti R (1999). Stereological assessment of the glial reaction to chronic deafferentation of the cochlear nuclei in the monkey (Macaca fascicularis). J Comp Neurology 414:485-94.

Valderrama-Canales FJ, Gil-Loyzaga P, Merchan Pérez A, Lopez Sanchez JG (1993). Astrocyte cytoarchitecture in cochlear nuclei of the rat: an immunocytochemical study. ORL 55:313-6.

West MJ, Østergaard K, Andreassen OA, Finsen B (1996). Estimation of the number of somatostatin neurons in the striatum: an in situ hybridization study using the optical fractionator method. J Comp Neurol 370:11-22. 